and pea tests. As these two compounds and (I) have many close chemical analogues, it is evident that the biological response is sensitive to very minute changes in the structure, or perhaps diffusibility, of the substances applied.

Eric Mitchell Crook.

Wriltam Davies.

Norma Ellzabeth Smith.

Botany and Chemistry Departments, University, Melbourne. Dec. 14.

${ }^{2}$ Ber., 68, 200 (1935).

- Proc. Acad. Sci. Amsterdam, 38, 896 (1935). Read in Chemical Abstracts, 30, 2600 (1936).

\section{Carotene Therapy of Retinitis Pigmentosa}

THE loss of dark adaptation and night blindness which characterizes the earliest stages of retinitis pigmentosa suggested to us the possibility that one of the fundamental features of the pathological physiology of the disease is failure of utilization of carotene and vitamin $\mathbf{A}$.

Feeding of these substances by mouth in large doses was found to have no effect upon the course of the disease or upon the symptoms in question. We thereupon administered carotene in oil intramuscularly in a series of cases, and were gratified to find a rapid and prompt response and relief of the night blindness. We administered carotene in doses of 30,000-60,000 New U.S.P. units intramuscularly, one to three times a week in our cases. In all the early cases of retinitis pigmentosa, improvement began to be noticeable within one weel after in. stitution of therapy; and dark adaptation became comparable to normal, and in some cases, normal within one to four months, with a corresponding improvement in the night blindness. In the more advanced cases with extensive chorio-retinal atrophy, no improvement has yet been noticed.

Our findings indicate that in retinitis pigmentosa there is a failure of assimilation of vitamin A and its precursors. We are planning a study of this phase of the subject. Another finding of interest is that, judging by the response of the cerebral circulation to bilateral carotid pressure-epileptiform convulsions and more or less prolonged loss of consciousness or coma-the arterial circulation of the brain of these cases is as much impaired as is that of the eye.

EMANUEL M. JosephSON. Moses Freiberger.

108 East Eighty-first Street, New York City.

Dec. 15.

\section{Diphenyl Compounds and Mammary Growth}

DodDs and Lawson ${ }^{1}$ have found that it is possible to obtain the ostrus response in castrated animals with substances which do not contain the phenanthrene nucleus. Various dihydroxydiphenyl com. pounds were tested and found to be active if the two hydroxyl groups were in the para position. Certain other compounds, for example, diphenyl- $\alpha$-naphthyl carbinol, were also found to be active.

Since these substances have cestrogenic properties, it might be expected that they would also function as initiators of mammary growth. Three compounds have been investigated for this effect, $p$ - $p^{\prime}$-dihydroxy diphenyl, 4-4'-dihydroxy-3-3'-dimethyl diphenyl, and diphenyl- $\alpha$-naphthyl carbinol. The compounds, suspended in sesame oil, were injected subcutaneously into young adult male guinea pigs, $100 \mathrm{mgm}$. being given once a week for three weeks.

With the diphenyl- $\alpha$-naphthyl carbinol, nipple growth commenced in a few days, and at the end of the three weeks the nipples had reached a size comparable to that at the end of the first pregnancy in the adult female. Histological examination revealed a limited duct growth, and several fairly extensive lobules of acinar tissue were present. Since the testicles were enlarged, and in some cases protruded through the abdominal wall, it is possible that the compound produced its effect through the endocrine system, and that the mammary response is perhaps due to increased secretion of testosterone. Collip, McEuen, and Selye ${ }^{2}$ have found that testosterone can cause the development of acinar tissue in the manmary glands of male animals; though Turner and Gomez ${ }^{3}$ state that œstrogenic hormones cannot cause growth of mammary parenchyma in completely hypophysectomized animals, the only effect observed being an extensive growth of the nipple. The answer to the question whether diphenyl- $\alpha$ naphthyl carbinol acts directly or indirectly in promoting mammary growth must therefore await the results of experiments with castrated and hypophysectomized animals.

In the doses given, the $p$ - $p^{\prime}$-dihydroxy diphenyl caused a slight but definite response, while the $4-4^{\prime}$ dihydroxy-3-3'-dimethyl diphenyl was inactive.

These observations were made during the progress of some experiments designed primarily to investigate the possible effect of the above compounds on lactose synthesis in the mammary gland.

Gordon Allison Gran'T

(Beit Memorial Research Fellow).

Department of Biochemistry, Lister Institute, London, S.W.1.

${ }^{1}$ Dodds and Lawson, NATure, 137, 996 (1936).

${ }^{2}$ Collip, McEuen and Selye, Amer. J. Physiol., 116, Proc., 29 (1936). Proc. Soc. Exp. Biol., N.Y., 34, 201 (1936).

${ }^{3}$ Gomez and Turner, Proc. Soc. Exp. Biol. N.Y., 34, 320 (1936).

\section{Temperature Variation of the Abnormal Unidirectional} Diamagnetism of Graphite Crystals

As is well-known, graphite crystal exhibits some remarkable magnetic properties. The susceptibility along the hexagonal axis of the crystal, $\chi_{11}$, is about $-22 \times 10^{-6}$ per gm., at room temperature, while that along directions in the basal plane, $\chi_{1}$, is $-0.5 \times 10^{-6}$ only ${ }^{1}$ - the latter value being nearly the same as that of diamond. The abnormal dia magnetism of graphite is thus more or less confined to one direction. Further, this abnormal diamagnetism is very sensitive to any chemical treatment of the crystal. For example, on treating the crystal with a mixture of strong nitric and sulphuric acids, when it swells up to 'blue graphite', $\chi_{\|}$ diminishes numerically from $-22 \times 10^{-8}$ to less than $-2 \times 10^{-6}$, whereas $\chi_{\perp}$ remains practically unchanged. Also an indefinite diminution in the size of the crystal appears to have the same effect.

We have recently studied the temperature variation of the principal susceptibilities of graphite, and the results may be of some interest in connexion with recent discussions on the contribution of 'free' electrons to diamagnetic susceptibility and its 